\title{
Divalproex in the Treatment of Impulsive Aggression: Efficacy in Cluster B Personality Disorders
}

\author{
Eric Hollander*,', Katherine A Tracy,3, Alan C Swann ${ }^{4}$, Emil F Coccaro ${ }^{5}$, Susan L McElroy ${ }^{6}$, Patricia \\ Wozniak $^{2}$, Kenneth W Sommerville ${ }^{2}$ and Charles B Nemeroff ${ }^{7}$ \\ 'Department of Psychiatry, Mount Sinai School of Medicine, New York, NY, USA; ${ }^{2}$ Abbott Laboratories, Abbott Park, IL, USA; ${ }^{3}$ Department of \\ Psychiatry, University of Illinois, Chicago, IL, USA; ${ }^{4}$ Department of Psychiatry, University of Texas Health Science Center, Houston, TX, USA; \\ ${ }^{5}$ Department of Psychiatry, University of Chicago/Pritzker School of Medicine, Chicago, IL, USA; ${ }^{6}$ Department of Psychiatry, University of Cincinnati \\ School of Medicine, Cincinnati, OH, USA; ' Department of Psychiatry and Behavioral Sciences, Emory University School of Medicine, Atlanta, GA, \\ USA
}

Impulsive aggressive behavior is common in psychiatric disorders and accounts for significant morbidity and mortality. However, little systematic treatment data exist from placebo-controlled trials for this symptom domain. This was a multicenter, randomized, doubleblind, placebo-controlled study in which outpatients with a score of $\geqslant 15$ on the Aggression scale of the Overt Aggression ScaleModified (OAS-M) and who fulfilled DSM-IV criteria for Cluster B personality disorder $(n=96)$, intermittent explosive disorder $(n=1 \mid 6)$, or post-traumatic stress disorder $(n=34)$ were randomized to divalproex sodium or placebo for 12 weeks duration. Based on average OAS-M Aggression scores over the last 4 weeks of treatment, a treatment effect was not observed in the intent-to-treat data set (combined across the three psychiatric disorders), but was observed in both intent-to-treat and evaluable data sets for patients with Cluster B personality disorders. In the Cluster B evaluable data set, statistically significant treatment differences favoring divalproex were also observed for component items of the OAS-M Aggression score, including verbal assault and assault against objects, as well as OASM Irritability score, and Clinical Global Impression (CGI)-Severity at multiple time points throughout the study. No treatment group difference was noted for overall premature discontinuation rate; however, across psychiatric diagnoses, 21 (17\%) patients in the divalproex group prematurely discontinued because of an adverse event, as compared to 4 (3\%) patients in the placebo group $(p<0.00 \mathrm{I})$. While a treatment effect was not observed when all diagnostic groups were combined, in a large subgroup of patients with Cluster B disorders, divalproex was superior to placebo in the treatment of impulsive aggression, irritability, and global severity. Neuropsychopharmacology (2003) 28, I I86-1 197, advance online publication, 2 April 2003; doi:10.1038/sj.npp.1300 I53

Keywords: divalproex; impulsive aggression; cluster B personality disorder

\section{INTRODUCTION}

Impulsive aggression is a significant public health problem that has received limited empirical attention (Hollander et al, 2002; Virkkunen, 1975; Pattison and Kahan, 1983; Cold, 1998). It has been defined by Coccaro and others as aggressive behavior (a verbal or physical act directed against a person or object that can potentially cause physical or emotional harm) that occurs in a deliberate

This study was supported by a grant from Abbott Laboratories, Abbott Park, IL. These data were presented in part at the 2002 Annual Meeting of the American Psychiatric Association, Philadelphia, PA, USA, May 18-23, 2002.

*Correspondence: Dr E Hollander, Department of Psychiatry, Mount Sinai School of Medicine, I Gustave L. Levy Place, Box 1230, New York, NY 10029-6574, USA, Tel: + I 212 24| 3623, Fax: + I 212987 403।,E-mail: eric.hollander@mssm.edu

Received 29 July 2002; revised 28 October 2002; accepted 07 January 2003

Online publication: 14 January 2003 at http://www.acnp.org/citations/ Npp0 | |40346 | and nonpremeditated fashion (Coccaro, 1998a; Moeller et al, 2001) and can be manifested by suicide attempts, self-injurious behavior, domestic violence, assault, and destruction of property. Many psychiatric disorders (eg, personality disorders, schizophrenia, bipolar disorder) are characterized by impulsive aggressive behavior, which often brings patients with psychiatric disorders to the attention of medical and forensic systems.

Numerous medications have been assessed for their efficacy in treating aggression in different diagnoses. Many of the studies in aggression had methodological limitations: patients were not diagnosed using operationalized criteria or structured interviews, placebo controls were lacking, impulsive aggression was not operationally defined or measured with validated or reliable instruments, and/or study samples were small. Moreover, treatment benefit was oftentimes mixed. With these methodological limitations in mind, preliminary data support the effectiveness of lithium, anticonvulsants, antipsychotics, and selective serotonin reuptake inhibitors (SSRIs) in decreasing aggression. Lithium has been shown to reduce aggression in children 
and adolescents with conduct disorder (Campbell et al, 1995; Malone et al, 2000), adults with borderline personality disorder (Links et al, 1990; Hori, 1998), and inmates without apparent psychiatric diagnoses (Tupin et al, 1973; Sheard et al, 1976). Phenytoin (in prison inmates) (Barratt et al, 1997) and carbamazepine (in personality disorder) (Cowdry and Gardner, 1988) have also been shown to decrease aggression. Standard antipsychotics have been used to decrease aggressive syndromes associated with borderline personality disorder, alcoholism/narcotic addiction, and cognitive impairment of aging (Itil and Wadud, 1975; Chengappa et al, 1999; Allain et al, 2000). Emerging evidence suggests that atypical antipsychotic agents may be helpful in impulsive aggression of borderline personality disorder (Khouzam and Donnelly, 1997; Zanarini and Frankenburg, 2001). Three double-blind, placebo-controlled studies have suggested that the SSRI fluoxetine decreases aggressive symptoms in patients with personality disorders (Fava et al, 1993; Salzman et al, 1995; Coccaro and Kavoussi, 1997).

A growing body of literature, including several doubleblind, placebo-controlled studies (Frankenberg and Zanarini, 2002; Hollander et al, 2001a; Tariot et al, 2001), supports a role for divalproex in the treatment of behavioral dyscontrol symptoms such as impulsivity, impulsive aggression, and hostility in a variety of psychiatric disorders. Divalproex improves symptoms of irritability and aggression in patients with personality disorders, in particular borderline personality disorder (Frankenberg et al, 2002; Hollander et al, 2001a; Kavoussi and Coccaro, 1998; Wilcox, 1995; Stein et al, 1995a). In addition, improvements have been noted following treatment with divalproex in patients with behavioral dyscontrol following traumatic brain injury (Horne and Lindley, 1995), temper outbursts (Donavan et al, 1997, 2000; Giakas et al, 1990), post-traumatic stress disorder (Petty et al, 2002; Fesler, 1991; Szymanski and Olympia, 1991), and agitation in the elderly (Tariot et al, 2001; Haas et al, 1997).

In this paper, we present the results of the first large, randomized, double-blind, placebo-controlled, multicenter study conducted to examine the efficacy and safety of divalproex in reducing the symptoms of impulsive aggression in patients with various psychiatric diagnoses. The entities of Cluster B personality disorder, intermittent explosive disorder, and post-traumatic stress disorder were chosen because they represent different types of diagnoses (ie, personality disorder, impulse control disorder, and anxiety disorder), but share a common symptomatology of impulsivity and aggression, which could benefit from the treatment. In this study, structured interviews were conducted to establish diagnoses, reliable instruments were used to assess aggression across different disorders, and the study was conducted over 3 months.

\section{PATIENTS AND METHODS}

\section{Patients}

Patients between 18 and 65 years of age with a diagnosis of Cluster B personality disorder (ie, antisocial, borderline, histrionic, and narcissistic personality disorders and cluster B personality disorder not otherwise specified [NOS]), intermittent explosive disorder, or post-traumatic stress disorder, as confirmed by the Structured Clinical Interview for DSM-IV conducted during screening (modified for this study by First et al, 1994), were selected for inclusion. If a patient met the criteria for more than one of these diagnoses, then the clinician at the investigative site had to specify as to which diagnosis was most closely associated with the symptoms of impulsive aggression in order for the patient to be randomized. A diagnosis of Cluster B personality disorder NOS required the presence of at least five features from more than one specific Cluster $B$ Personality Disorder, and full criteria for any one Cluster B Personality Disorder were not met.

Patients were required to have, on average, two episodes of physical or verbal aggressive outbursts per week for at least the month prior to screening, causing marked distress or impairment in occupational or interpersonal function. The aggressive behavior was judged to be neither premeditated nor committed to achieve a tangible objective (Coccaro et al, 1998b). Patients must also have had a minimum score of 15 on the Aggression scale (items 1-4) of the Overt Aggression Scale-Modified for outpatients (OASM) (Yudofsky et al, 1986; Coccaro et al, 1991) at the first screening visit and at either the second screening visit or at randomization. Patients receiving psychotherapy must have had a stable psychotherapy schedule for at least 3 months prior to screening that was maintained throughout the study.

Patients were excluded from the study if they had any of the following psychiatric disorders: lifetime bipolar I disorder, bipolar II disorder with hypomania in the past year or a baseline Mania Syndrome Scale Score of $\geqslant 12$, major depressive disorder of significant severity (score $>15$ on the 17-item Hamilton Psychiatric Rating Scale for Depression [HAM-D]), history of schizophrenia or other psychotic disorder, or symptoms of dementia. Patients with serious homicidal or suicidal ideation were also excluded from the study, as were patients with impulsive aggression that resulted from previous head trauma or other medical condition, pregnant or lactating females, and patients with clinically significant abnormal laboratory data, unstable medical conditions, or any underlying condition that would confound the interpretation of study results.

Patients were to avoid concurrent use of any psychotropic medication other than the study drug during the study, with a few exceptions. The exceptions included SSRIs (eg, fluoxetine, sertraline), tricyclic antidepressants (eg, amitriptyline, desipramine), and stimulants (eg, methylphenidate), only when patients had taken a stable dose of the allowable psychotropic medication for at least 2 months prior to screening and continued its use at the same dose throughout the study. Patients were also allowed zolpidem tartrate (up to $10 \mathrm{mg} /$ day) for up to 4 days per week, but not within $8 \mathrm{~h}$ prior to efficacy ratings, for the control of insomnia. Patients were specifically prohibited from use of benzodiazepines, mood stabilizers/anticonvulsants, monoamine oxidase inhibitors (MAOI), or antipsychotic agents.

\section{Study Design}

The study was a randomized, double-blind, placebocontrolled, parallel-group, multicenter (19 sites) trial, consisting of a screening period not to exceed 14 days, a 
12-week double-blind treatment period, and a 1-week tapering period. The protocol was approved by the institutional review board of each participating study site. Written informed consent was obtained from each patient or the patient's legally authorized representative before enrollment into the study.

Patients were randomized in equal numbers, within each of the three diagnostic groups, to receive either divalproex sodium delayed-release tablets (Depakote ${ }^{\circledR}$, Abbott Laboratories, Abbott Park, IL) or matching placebo. Divalproex was initiated at $500 \mathrm{mg} /$ day (administered twice daily) and was increased by $250 \mathrm{mg}$ every 3-7 days during the first 3 weeks of treatment. The dose and titration schedule of study drug for each patient was determined by their investigator and was based on the patient's clinical response and tolerance of study drug. The recommended valproate serum level was $80-120 \mu \mathrm{g} / \mathrm{ml}$ by week 3 , and the maximum dose was $30 \mathrm{mg} / \mathrm{kg} /$ day.

Trough serum concentrations of valproate (samples collected prior to dosing) were determined at weeks 3,6 , and 12. An unblinded person from the central laboratory reported serum valproate levels of $<80 \mu \mathrm{g} / \mathrm{ml}$ or $>120 \mu \mathrm{g} /$ $\mathrm{ml}$ to the investigators, so that the dose of study drug could be adjusted appropriately. In order to preserve the study blind, sham valproate levels were reported for selected placebo patients.

After patients completed the 12 -week course of study drug or prematurely discontinued participation in the study, their dosage of study drug was reduced (tapered) to zero, at the discretion of the investigators, within a 7-day period. An alternative drug regimen of the investigators' choosing could be initiated during the study drug tapering period.

\section{Clinical Evaluations}

Protocol-defined psychiatric assessments included the OAS$\mathrm{M}$ and the Clinical Global Impression (CGI) Scale (Guy, 1976). The OAS-M is comprised of an Aggression score, which is the sum of individual items of verbal assault, assault against objects, assault against others, and assault against self. The OAS-M also assesses irritability (sum of two items) and suicidal tendencies (one item). The OAS-M evaluation was conducted at baseline and weekly thereafter, with telephone visits at weeks 5 and 7. The CGI evaluation (focused on global psychopathology, not solely aggression) was conducted at baseline and once a week, excluding weeks 5 and 7.

All ratings were performed by trained and qualified personnel, and, if possible, all ratings were performed by the same individual for a given patient. For the OAS-M, raters were considered qualified if their scores from mock patient interviews were within $95-105 \%$ of a predetermined score. An assessment of inter-rater reliability was conducted during the trial (30 raters from 16 sites) to assure the proficiency of the raters; inter-rater reliability was high $(\mathrm{ICC}=0.96)$ (Endicott et al, 2002).

\section{Safety Assessment}

The data obtained to evaluate the safety of study drug included physical examinations, adverse events, and laboratory test results. Patients were monitored for adverse events between the time study drug was initiated and up to 30 days after the discontinuation of therapy, inclusive.

An independent Data and Safety Monitoring Board (DSMB) was established for this study. The DSMB was comprised of psychiatrists, an internist/epidemiologist, and statisticians. Under blinded conditions, the DSMB assessed the ongoing safety of interventions to patients in this study. The DSMB reviewed the data on patient demographics, aggression history, occurrence of acts of aggression that were dangerous to the patient or others, adverse events, treatment discontinuations, and abnormal laboratory values. Based on their review, the DSMB was to make recommendations, if necessary, concerning premature termination of the study, changes in the study protocol, or changes in the informed consent form. Based on the input of the DSMB, the study protocol was amended to exclude patients under a restraining order or order of protection and to explicitly allow for removing patients from the study if they were deemed to be at significant risk of hurting themselves or others. In addition, following DSMB recommendations, an aftercare program was made available whereby patients could opt for limited aftercare (eg, medication, psychotherapy) following participation in the study.

\section{Statistical Analyses}

The primary objective of this study was to evaluate the efficacy and safety of divalproex in the treatment of impulsive aggression in patients who satisfied the diagnostic criteria for Cluster B personality disorder, posttraumatic stress disorder, or intermittent explosive disorder. The primary efficacy end point was the average of the evaluations obtained during the last 4 weeks of treatment for the OAS-M Aggression score in the intent-to-treat data set that combined all three psychiatric diagnoses. Secondary efficacy end points included: (1) the average of the evaluations obtained during the last 4 weeks of treatment for the OAS-M Aggression score in the evaluable data set that combined all three psychiatric diagnoses; (2) the average of the evaluations obtained during the last 4 weeks of treatment for the OAS-M Aggression score in intent-totreat and evaluable data sets by psychiatric diagnosis; and (3) change from baseline to each evaluation time point for OAS-M Aggression score and its component items, irritability, and suicidal tendencies in intent-totreat and evaluable data sets that combined all three psychiatric diagnoses and in intent-to-treat and evaluable datasets by psychiatric diagnosis. All CGI evaluations were secondary end points, with the exception of percent treatment responders at week 12, which was a post hoc analysis.

All statistical tests were two-tailed, and $p$-values of 0.050 , after rounding to three decimal places, were considered statistically significant.

Efficacy analyses were performed using an intent-to-treat data set and an evaluable data set. The intent-to-treat data set included all patients who received at least one dose of study drug and had at least one on-treatment OAS-M rating. The evaluable patients data set was defined a priori and excluded all data for patients who were treated for less than 
21 days. In addition, for those patients with a change in a protocol-allowed psychotropic medication dose, data obtained after the change in dose were excluded from the evaluable data set. Exclusions from the evaluable data set were determined before the study blind was broken. To address missing evaluations, a last observation carried forward (LOCF) analysis was conducted. This technique was used to reduce bias caused by patients who prematurely discontinued for lack of efficacy.

Baseline comparability between the treatment groups for demographic characteristics was assessed by one-way analysis of variance (ANOVA) with treatment group as the main effect for quantitative variables (ie, age, weight) and by Fisher's exact test for qualitative variables (ie, gender, race). For statistical testing, race was categorized as Caucasian and non-Caucasian. For psychiatric history variables, baseline comparability between treatment groups was assessed by the Wilcoxon rank sum test (age at first major depressive episode), by the Cochran-Mantel-Haenszel test (total number of prior major depressive episodes and lifetime number of psychiatric hospitalizations), and by Fisher's exact test (family history of psychiatric illness, trauma history, history of addictive behavior, and history of prosecution). Baseline comparability between treatment groups for OAS-M Aggression score and Irritability score was assessed by van Elteren analyses (ie, Wilcoxon ranksum test stratified by diagnosis).

Treatment differences in the percentage of patients using zolpidem were assessed by Fisher's exact test. Treatment differences in the number and percentage of days zolpidem was prescribed and in the average daily dose of zolpidem were evaluated by Wilcoxon rank-sum test.

OAS-M item scores had a minimum value of zero, but no upper limit on the maximum value, which resulted in highly skewed actual scores and change in scores. Therefore, van Elteren analyses stratified by diagnosis and Wilcoxon analyses were performed to reduce the effect of outliers on the results, and median values were reported for OAS-M data. For the OAS-M Aggression score and its component items, irritability and suicidal tendencies, treatment differences for the median average of the evaluations obtained during the last 4 weeks of treatment as well as for the change from baseline to each evaluation time point were analyzed using van Elteren analyses stratified by diagnosis (for all patients) and the Wilcoxon rank-sum test (for analyses by diagnostic subgroup). Analyses of treatment differences in the mean change from baseline to each evaluation among Cluster B patients were conducted using a one-way ANOVA with treatment as the main effect for CGI-Severity score, and using the Cochran-Mantel-Haenszel test for CGIImprovement score.

Safety analyses were performed for all patients who received at least one dose of randomized study drug. Fisher's exact test was used to assess treatment group differences in treatment-emergent adverse event incidence rates. Treatment differences in the percentage of patients prematurely discontinuing from the study were assessed by Fisher's exact test both overall and for each reason.

Given the planned sample size of 120 patients in each study drug group and a type I error of 0.05 for a two-sided test, the study had 80 and $90 \%$ power to detect effect sizes of 0.36 and 0.42 , respectively.

\section{RESULTS}

\section{Patient Disposition}

In all, 246 patients were randomized and received at least one dose of study drug; 96 patients (47 divalproex and 49 placebo) had a primary diagnosis (the disorder primarily associated with impulsive aggressive symptoms) of Cluster B personality disorder, 116 patients (59 divalproex and 57 placebo) had a primary diagnosis of intermittent explosive disorder, and 34 patients (18 divalproex and 16 placebo) had a primary diagnosis of post-traumatic stress disorder. Of the 246 patients who received study drug, 233 (116 and 117 patients in the divalproex and placebo groups, respectively) were included in the intent-to-treat analyses of efficacy and 13 patients were excluded because they did not have a post-baseline OAS-M Aggression score.

Of the 96 randomized patients with a Cluster B personality disorder, 91 patients (43 divalproex and 48 placebo) were included in the intent-to-treat data set; the most common primary diagnosis was borderline personality disorder (55\% of patients), followed by Cluster B personality disorder NOS (21\%), narcissistic (13\%), antisocial $(10 \%)$, and histrionic $(1 \%)$ personality disorders. The evaluable data set for analyses of efficacy included 85 patients (39 divalproex and 46 placebo). Five patients (four patients in the divalproex group and one patient in the placebo group) were excluded from the intent-to-treat analyses because they were lacking a post-baseline OAS-M Aggression score. Six additional patients were excluded from the evaluable data set for all efficacy analyses because they had taken the study drug for less than 21 days (four patients in the divalproex group and two patients in the placebo group). Two additional patients in the placebo group had partial data excluded (from study days 66-80 for one and study days 57-85 for the other) because they changed doses of protocol-allowed psychotropic medications (ie, sertraline and amitriptyline).

\section{Baseline Demographic and Clinical Characteristics}

The treatment groups were similar at baseline based on demographics, history of major depressive disorder, number of past psychiatric hospitalizations, and histories of trauma, addictive behavior, and prosecution (Table 1). The mean age of the intent-to-treat study population was 40.3 years (range, 19-67 years). The majority was male (73\%) and Caucasian $(84 \%)$. A third $(33 \%)$ had a past history of at least one major depressive episode prior to study enrollment. Only $15 \%$ of patients had been hospitalized for psychiatric indications prior to enrollment in the study. A total of $58 \%$ of patients reported a history of trauma, with about a third (31\%) of patients reporting physical abuse and $14 \%$ reporting sexual abuse. Prior histories of alcohol (32\% of patients) and drug ( $16 \%$ of patients) abuse/dependence were also reported. About half $(46 \%)$ had been arrested. The median (mean) baseline OAS-M Aggression score was 43.7 (66.7) and 33.7 (66.5) for patients in the divalproex and placebo groups, respectively, with no significant difference between treatment groups. Baseline demographic and clinical characteristics of the Cluster B patients were generally similar to those of the overall population (mean age of 37.3 years, $63 \%$ male). 
Table I Baseline Demographic and Clinical Characteristics of Intent-to-Treat Patients

\begin{tabular}{|c|c|c|}
\hline Characteristic & Placebo $(n=117)$ & Divalproex $(n=116)$ \\
\hline \multicolumn{3}{|l|}{ Gender, n (\%) } \\
\hline Female & $37(32 \%)$ & $27(23 \%)$ \\
\hline Male & $80(68 \%)$ & $89(77 \%)$ \\
\hline \multicolumn{3}{|l|}{ Race } \\
\hline Caucasian & $100(85 \%)$ & $95(82 \%)$ \\
\hline Black & $10(9 \%)$ & $16(14 \%)$ \\
\hline Other & $7(6 \%)$ & $5(4 \%)$ \\
\hline \multicolumn{3}{|l|}{ Age (years) } \\
\hline Mean $( \pm S D)$ & $39.7(11.55)$ & $41.0(11.80)$ \\
\hline Range & $20-67$ & $19-64$ \\
\hline \multicolumn{3}{|l|}{ Weight (lb) } \\
\hline Mean $( \pm S D)$ & $186.5(52.91)$ & $188.0(38.48)$ \\
\hline Range & $110-400$ & $100-283$ \\
\hline \multicolumn{3}{|l|}{ Total no. of prior major depressive episodes } \\
\hline 0 & $77(66 \%)$ & $78(67 \%)$ \\
\hline $\mid-5$ & $32(27 \%)$ & $31(27 \%)$ \\
\hline$>5$ & 8 (7\%) & 7 (6\%) \\
\hline \multicolumn{3}{|l|}{ Age at first major depressive episode (years) } \\
\hline Mean $( \pm S D)$ & $26.6(11.7)$ & $27.7(11.8)$ \\
\hline Range & 8-52 & $10-52$ \\
\hline \multicolumn{3}{|l|}{ Lifetime number of psychiatric hospitalizations } \\
\hline Never & 99 (85\%) & $98(84 \%)$ \\
\hline $1-5$ & $16(14 \%)$ & $18(16 \%)$ \\
\hline$>6$ & $2(2 \%)$ & $0(0 \%)$ \\
\hline Family history of psychiatric illness & $19(16 \%)$ & $24(21 \%)$ \\
\hline \multicolumn{3}{|l|}{ Trauma history } \\
\hline Any trauma history & $62(54 \%)$ & $70(61 \%)$ \\
\hline Physical abuse trauma history & $35(30 \%)$ & $38(33 \%)$ \\
\hline Sexual abuse trauma history & $16(14 \%)$ & $16(14 \%)$ \\
\hline \multicolumn{3}{|l|}{ History of addictive behavior } \\
\hline History of alcohol abuse/dependence & $38(32 \%)$ & 37 (32\%) \\
\hline History of drug abuse/dependence & $17(15 \%)$ & $21(18 \%)$ \\
\hline \multicolumn{3}{|l|}{ History of prosecution } \\
\hline History of arrest & $53(45 \%)$ & $55(47 \%)$ \\
\hline History of incarceration & $21(18 \%)$ & 31 (27\%) \\
\hline Median OAS-M Aggression score & 33.7 & 43.7 \\
\hline Median irritability score & 6.3 & 6.3 \\
\hline
\end{tabular}

Note: $p>0.05$ for all comparisons.

A small proportion of study patients used an antidepressant during the treatment period (21 [17\%] of divalproex-treated patients and 19 [16\%] of placebo-treated patients). The use of zolpidem, which was permitted by the protocol for the alleviation of insomnia, was similar between the treatment groups, including the percentage of patients using zolpidem, the number of days used, and the mean daily dose. A total of 15 divalproex-treated patients (13\%) and nine placebo-treated patients (8\%) used zolpidem at least once during their participation in the study.

\section{Effect of Divalproex on Aggression in Patients with Psychiatric Disorders}

Across the psychiatric disorders, there was no significant treatment difference between divalproex and placebo based on median average of OAS-M Aggression scores obtained during the last 4 weeks of treatment in the intent to-treat population $\left(\chi_{(1)}^{2}=0.000, p=0.989\right)$ (Table 2). There was no evidence of heterogeneity across sites. When the data were analyzed by primary diagnosis, an antiaggressive effect for divalproex was observed among the patients with a primary diagnosis of a Cluster B personality disorder, but not intermittent explosive disorder or post-traumatic stress disorder. The balance of this paper will focus on the analyses that were conducted in the Cluster B personality disordered patients.

\section{Patients with Cluster B Personality Disorders}

Effect of divalproex on aggression. According to the intentto-treat analysis, there was a significant treatment difference favoring divalproex over placebo based on the primary end point, the median average of OAS-M Aggression scores obtained during the last 4 weeks of treatment $\left(\chi_{(1)}^{2}=3.952\right.$, $p=0.047)$ (Table 3$)$. More than half of patients treated with divalproex had an average OAS-M Aggression score during the final 4 weeks of treatment that would have made them ineligible for enrollment into the study. There were trends on the subscales of the Aggression score for verbal assault $(p=0.060)$ and assault against others $(p=0.068)$, but not for assault against objects $(p=0.134)$ or assault against self $(p=0.222)$.

For the evaluable data set, treatment with divalproex resulted in statistically significantly lower (better) average 
Table 2 Baseline and Average of Last 4 Weeks for OAS-M Aggression Scores in Intent-toTreat Patients with a History of Impulsive Aggressive Behavior

\begin{tabular}{|c|c|c|c|c|}
\hline & Placebo & Divalproex & $\chi_{(1)}^{2}$ & $p-$ Value $^{\mathrm{a}}$ \\
\hline \multicolumn{5}{|l|}{ All patients } \\
\hline$N$ & 117 & 116 & & \\
\hline \multicolumn{5}{|l|}{ Baseline $^{b}$} \\
\hline Median & 33.7 & 43.7 & 1.552 & 0.213 \\
\hline Mean \pm SD & $62.3 \pm 88.8$ & $63.3 \pm 61.8$ & & \\
\hline \multicolumn{5}{|c|}{ Average of last 4 weeks } \\
\hline Median & 12.3 & 10.6 & 0.000 & 0.989 \\
\hline Mean \pm SD & $32.1 \pm 57.2$ & $34.5 \pm 71.3$ & & \\
\hline \multicolumn{5}{|c|}{ Intermittent explosive disorder } \\
\hline N & 54 & 55 & & \\
\hline \multicolumn{5}{|l|}{ Baseline $^{b}$} \\
\hline Median & 30.0 & 44.0 & 2.474 & 0.116 \\
\hline Mean \pm SD & $65.5 \pm 114.3$ & $62.0 \pm 65.8$ & & \\
\hline \multicolumn{5}{|c|}{ Average of last 4 weeks } \\
\hline Median & 9.0 & 13.0 & 2.580 & 0.108 \\
\hline Mean \pm SD & $28.9 \pm 59.9$ & $28.9 \pm 39.1$ & & \\
\hline \multicolumn{5}{|c|}{ Cluster B personality disorder } \\
\hline N & 48 & 43 & & \\
\hline \multicolumn{5}{|l|}{ Baseline $^{b}$} \\
\hline Median & 35.2 & 35.7 & 0.003 & 0.956 \\
\hline Mean \pm SD & $54.8 \pm 56.3$ & $54.9 \pm 48.8$ & & \\
\hline \multicolumn{5}{|c|}{ Average of last 4 weeks } \\
\hline Median & 16.3 & 8.3 & 3.952 & 0.047 \\
\hline Mean \pm SD & $38.6 \pm 61.1$ & $29.2 \pm 66.1$ & & \\
\hline \multicolumn{5}{|c|}{ Post-traumatic stress disorder } \\
\hline N & 15 & 18 & & \\
\hline \multicolumn{5}{|l|}{ Baseline $^{b}$} \\
\hline Median & 45.7 & 50.2 & 0.294 & 0.588 \\
\hline Mean \pm SD & $74.5 \pm 69.9$ & $86.9 \pm 73.9$ & & \\
\hline \multicolumn{5}{|c|}{ Average of last 4 weeks } \\
\hline Median & 14.3 & 14.9 & 0.173 & 0.678 \\
\hline Mean \pm SD & $22.9 \pm 27.1$ & $64.2 \pm 132.6$ & & \\
\hline
\end{tabular}

${ }^{a}$ OAS-M item scores had a minimum value of zero, but no upper limit on the maximum value, which resulted in highly skewed actual scores and change in scores. Therefore, van Elteren analyses were conducted for all patients and Wilcoxon rank-sum test was performed for diagnostic subgroups comparing median average of OAS-M scores over the last 4 weeks of treatment.

${ }^{\mathrm{b}}$ Average of evaluations conducted prior to dosing.

scores over the last 4 weeks of treatment compared to placebo for the OAS-M Aggression score $\left(\chi_{(1)}^{2}=5.779\right.$, $p=0.016)$, the OAS-M verbal assault subscale $\left(\chi_{(1)}^{2}=4.520\right.$, $p=0.034)$, assault against objects subscale $\left(\chi_{(1)}^{2}=3.982\right.$, $p=0.046)$, and assault against others subscale $\left(\chi_{(1)}^{2}=4.333\right.$, $p=0.037$ ) (Table 3). Scores on the OAS-M assault against self subscale, both prior to and during the study, were too low for meaningful comparisons. Improvements from baseline to scheduled evaluations of the OAS-M Aggression score and its component items were observed throughout the 12-week study for both treatment groups, with statistically significant differences between treatment groups $(p<0.05)$ favoring divalproex at week 12 for OAS$M$ Aggression score (Figure 1) and OAS-M verbal assault subscale.

Effect of divalproex on irritability. Among evaluable Cluster B patients, the median average score over the last 4 weeks of treatment for OAS-M Irritability was significantly lower (improved) for divalproex, compared with the average score for placebo $\left(3.5\right.$ vs $4.4, \chi_{(1)}^{2}=4.545, p=0.033$ (baseline median of 6.3 for both groups). Throughout the 12-week study, improvements from baseline to scheduled evaluations were observed for OAS-M Irritability score in both treatment groups, with statistically significant differences between treatment groups $(p<0.05)$ favoring divalproex at multiple time points (Figure 2).

Effect of divalproex on CGI ratings. Improvements from baseline in CGI-Severity (CGI-S) score were similar (parallel) to those observed for Irritability, and greater with divalproex than with placebo at all evaluation time points, with differences reaching the level of statistical significance $(p<0.05$, one-way ANOVA) at weeks $1,3,6$, and 9-12 among evaluable patients (Figure 3 ). According to analyses of CGI-Improvement (CGI-I) scale (eg $1=$ very much improved, $2=$ much improved, $3=$ minimally improved) in evaluable patients, a treatment effect was observed for mean CGI-I score at week 3 (2.3 with divalproex $v s 2.9$ with placebo, $\chi_{(1)}^{2}=4.942, p=0.026$ by the Cochran-Mantel- 
Table 3 Median Baseline and Median Average of Last 4 Weeks for OAS-M Scores in Patients with Cluster B Personality Disorders and History of Impulsive Aggressive Behavior

\begin{tabular}{|c|c|c|c|c|c|c|}
\hline \multirow[b]{2}{*}{ OAS-M subscale } & \multicolumn{2}{|c|}{ Baseline $^{a}$} & \multicolumn{2}{|c|}{ For last 4 weeks } & \multirow[b]{2}{*}{$\chi_{(1)}^{2}$} & \multirow[b]{2}{*}{$p$-Value } \\
\hline & Placebo & Divalproex & Placebo & Divalproex & & \\
\hline \multicolumn{7}{|l|}{ Intent-to-treat data set ${ }^{\mathrm{c}}$} \\
\hline Aggression score & 35.2 & 35.7 & 16.3 & 8.3 & 3.952 & 0.047 \\
\hline Verbal assault & 25.7 & 22.3 & 10.8 & 6.0 & 3.536 & 0.060 \\
\hline Assault against objects & 2.3 & 2.0 & 0.5 & 0.3 & 2.250 & 0.134 \\
\hline Assault against others & 1.3 & 1.3 & 0.3 & 0.0 & 3.331 & 0.068 \\
\hline Assault against self & 0.0 & 0.0 & 0.0 & 0.0 & 1.492 & 0.222 \\
\hline \multicolumn{7}{|l|}{ Evaluable data set ${ }^{\mathrm{d}}$} \\
\hline Aggression score & 33.6 & 34.3 & 16.3 & 6.8 & 5.779 & 0.016 \\
\hline Verbal assault & 25.0 & 22.3 & 10.8 & 6.0 & 4.520 & 0.034 \\
\hline Assault against objects & 2.3 & 2.0 & 0.5 & 0.3 & 3.982 & 0.046 \\
\hline Assault against others & 1.3 & 1.3 & 0.3 & 0.0 & 4.333 & 0.037 \\
\hline Assault against self & 0.0 & 0.0 & 0.0 & 0.0 & 1.254 & 0.263 \\
\hline
\end{tabular}

${ }^{a}$ Average of evaluations conducted prior to dosing.

based on Wilcoxon rank-sum test comparing average of OAS-M scores over the last 4 weeks of treatment.

${ }^{c} n=43$ for divalproex and $n=48$ for placebo.

${ }^{d} n=39$ for divalproex and $n=46$ for placebo.

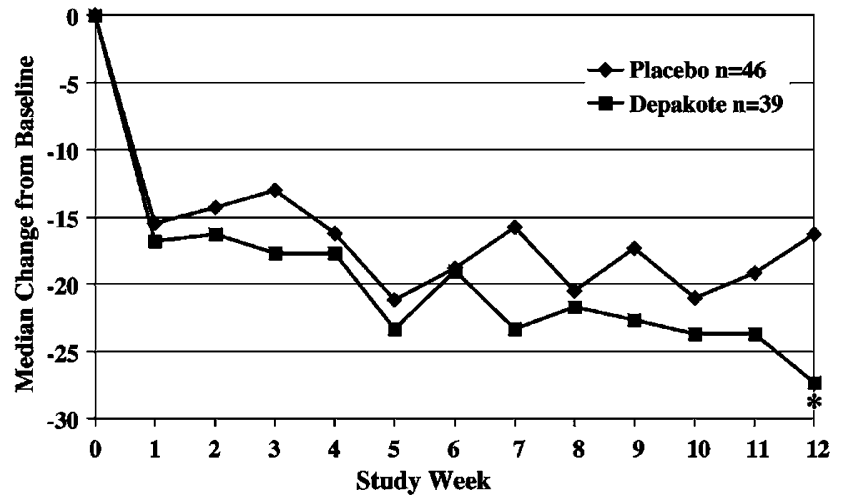

Baseline: PBO = 33.6 Dep $=34.3$

Figure I Median change from baseline OAS-M Aggression score in patients with Cluster B personality disorders and history of impulsive aggressive behavior.

Haenszel test), week 11 (2.1 vs 2.6, respectively; $\chi_{(1)}^{2}=3.880$, $p=0.049)$, and week 12 (2.1 vs 2.7 , respectively; $\chi_{(1)}^{2}=5.478$, $p=0.019$ ). At week 12 , significantly more of the divalproextreated Cluster B patients (72\%) were rated as treatment responders (a score of 1 or 2 on the CGI-I), compared to the placebo-treated patients $(50 \%)(p=0.035)$.

Dosing of divalproex. The mean duration of exposure to divalproex was 63 days and the mean modal daily dose of divalproex was $1567 \mathrm{mg}$ (range, 500-3000 mg), resulting in a mean trough valproate serum level of $64.2 \mu \mathrm{g} / \mathrm{ml}$ (range, $0.0-147 \mu \mathrm{g} / \mathrm{ml}) \quad(n=102)$ at the final evaluation in all divalproex-treated patients. For patients in the Cluster B group, the mean duration of exposure to divalproex was 62 days and the mean modal daily dose of divalproex was $1404 \mathrm{mg}$ (range, $500-2250 \mathrm{mg}$ ), resulting in a mean trough valproate serum level of $65.5 \mu \mathrm{g} / \mathrm{ml}$ (range, $0.0-147 \mu \mathrm{g} / \mathrm{ml}$ ) $(n=39)$ at the final evaluation.

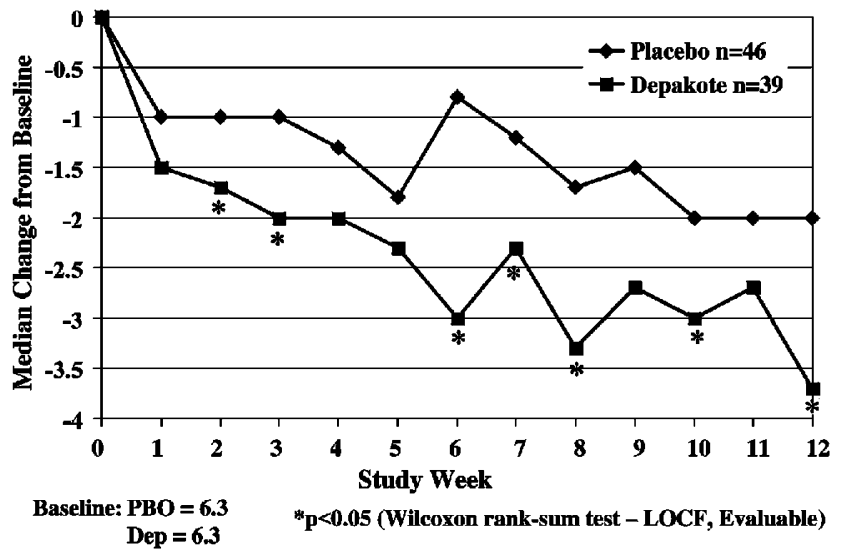

Figure 2 Median change from baseline OAS-M Irritability score in patients with Cluster B personality disorders and history of impulsive aggressive behavior.

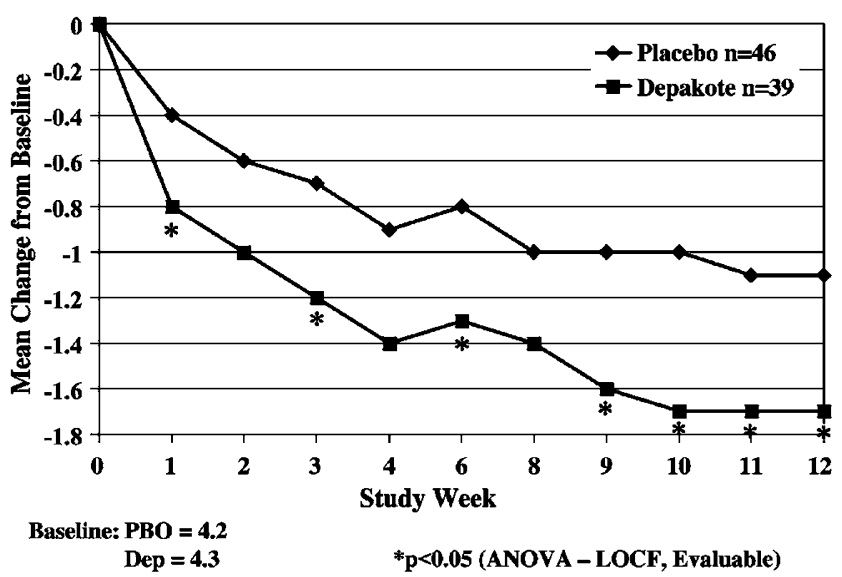

Figure 3 Mean change from baseline CGI-Severity score in patients with Cluster B personality disorders and history of impulsive aggressive behavior. 
Table 4 Treatment Emergent Adverse Events ${ }^{\text {a }}$ Occurring in $\geqslant 10 \%$ of Patients in any Treatment Group or that were Statistically Significantly Different Between Groups

\begin{tabular}{lcc}
\hline & Placebo (n= I 22) & Divalproex (n= I24) \\
\hline Any adverse event & $99(81 \%)$ & $115(93 \%)^{\mathrm{b}}$ \\
Somnolence & $29(24 \%)$ & $42(34 \%)$ \\
Headache & $34(28 \%)$ & $26(21 \%)$ \\
Nausea & $15(12 \%)$ & $35(28 \%)^{\mathrm{b}}$ \\
Infection & $26(21 \%)$ & $16(13 \%)$ \\
Diarrhea & $15(12 \%)$ & $26(21 \%)$ \\
Dyspepsia & $15(12 \%)$ & $20(16 \%)$ \\
Asthenia & $10(8 \%)$ & $23(19 \%)^{\mathrm{b}}$ \\
Insomnia & $14(11 \%)$ & $17(14 \%)$ \\
Dizziness & $10(8 \%)$ & $16(13 \%)$ \\
Weight gain & $7(6 \%)$ & $17(14 \%)$ \\
Abdominal pain & $9(7 \%)$ & $14(11 \%)$ \\
Vomiting & $6(5 \%)$ & $14(11 \%)$ \\
Increased appetite & $3(2 \%)$ & $13(10 \%)^{\mathrm{b}}$ \\
Depression & $3(2 \%)$ & $12(10 \%)^{\mathrm{b}}$ \\
SGPT increased & $1(<1 \%)$ & $14(11 \%)^{\mathrm{b}}$ \\
Tremor & $0(0 \%)$ & $15(12 \%)^{\mathrm{b}}$ \\
SGOT increased & $1(<1 \%)$ & $13(10 \%)^{\mathrm{b}}$ \\
Nervousness & $0(0 \%)$ & $9(7 \%)^{\mathrm{b}}$ \\
\hline
\end{tabular}

In decreasing order of overall occurrence.

${ }^{b} p<0.05$ for divalproex vs placebo.

\section{Safety and Premature Discontinuations From the Study}

Among the 246 randomized patients (across psychiatric diagnoses) who received the study drug, a higher proportion of patients in the divalproex group reported at least one treatment-emergent adverse event $(p=0.008)$ (Table 4$)$. Most adverse events were mild or moderate in severity. The most commonly reported adverse event was somnolence (29\% of patients), followed by headache $(24 \%)$ and nausea (20\%). The difference between treatment groups in incidence of a specific treatment-emergent adverse event was statistically significant for asthenia, depression, increased appetite, increases in SGPT and SGOT, nausea, nervousness, and tremor, with a higher incidence for divalproex.

Three patients in the divalproex group (depression and hostility, cerebrovascular accident, and anxiety) and two patients in the placebo group (supraventricular tachycardia and pericarditis) experienced at least one adverse event that was considered serious. None of the serious adverse events was classified by the investigator as related to the study drug.

In all, 101 patients (54/124 [44\%] and 47/122 [39\%] of divalproex and placebo-treated patients, respectively) prematurely discontinued their participation in the study. The reasons for early dropouts were treatment-emergent adverse events (21 and four patients in the respective treatment groups) $(p<0.001)$, lost to follow-up (12 and 13 patients, respectively), noncompliance (seven and 11 patients, respectively), ineffectiveness (four and six patients, respectively), and other reasons (10 and 13 patients, respectively; eg, personal reasons, patient required excluded medications or a change to allowed medications, patient withdrew consent, site error). The most common adverse events leading to premature discontinuation of study drug included abnormal laboratory values, depression, nausea, and vomiting. Increased liver function tests, which the investigator considered an adverse event, contributed to the premature discontinuation of three patients in the divalproex group and two patients in the placebo group. Hyperkalemia (divalproex), hyperglycemia (divalproex), and hyperuricemia (placebo) contributed to the premature discontinuation for one patient each.

In Cluster B patients, the type and distribution of adverse events were generally similar to that observed among all treated patients. There was no difference between treatment groups for overall premature discontinuation rate in patients with Cluster B personality disorders $(47 \%$ and $45 \%$ of divalproex and placebo-treated patients, respectively). Eight (17\%) patients in the divalproex group discontinued their participation in the study because of a treatment-emergent adverse event, as did two (4\%) patients in the placebo group $(p=0.048)$. The pattern of adverse events leading to premature discontinuation of Cluster B patients was similar to that for the total population.

\section{DISCUSSION}

These data represent the first large, multicenter, doubleblind, placebo-controlled trial conducted to evaluate treatment of impulsive aggression. This study utilized a novel approach by examining divalproex in the treatment of symptoms of impulsive aggression in patients with Cluster B personality disorder, intermittent explosive disorder, or post-traumatic stress disorder. The current study differs from the previous small trials either in utilizing the OAS-M or including patients with much higher baseline scores on OAS-M and in examining results across multiple centers.

While a treatment effect was not observed based on the primary end point (average of OAS-M Aggression scores obtained during the last 4 weeks of treatment for all diagnostic groups combined), analyses on diagnostic subsets of the data revealed clinically important findings. Divalproex improved impulsive aggressive behavior in patients with Cluster B personality disorders as assessed by the OAS-M Aggression score at end point and at week 12. Similar findings were not observed in the intermittent explosive and post-traumatic stress disorder groups, in which 70 and 69\% decreases in OAS-M Aggression score were observed in the respective placebo groups. The divalproex response (77\%) was comparable in the Cluster B group, but the placebo response was only 54\%. These data raise the possibility that impulsive aggression may capture a biologically heterogeneous phenomenon and that there may be subgroups within the broad category of impulsive aggression that are homogeneous in their sensitivity to divalproex.

In the Cluster B evaluable subset, treatment differences were observed on the OAS-M Aggression and on the OAS-M items of verbal assault, assault against objects, and assault against others. These findings suggest that future research consider excluding patients who have not had adequate exposure to study medication. Cluster B patients who comply with at least 3 weeks of treatment may be more likely to benefit from potential antiaggressive effects of divalproex. In addition to an antiaggressive effect, a significant treatment difference favoring divalproex was 
observed early in the treatment for OAS-M Irritability and for global measures of CGI-S. This treatment effect remained fairly consistent throughout the duration of the trial. While modest, the temporal relation suggests that the effect of divalproex on irritability may contribute to the treatment effect for impulsive aggression. The data on the Cluster B personality disorder subgroup should be interpreted cautiously as these findings were demonstrated in secondary analyses.

No unexpected safety concerns were identified during the course of this study. Adverse events were generally mild or moderate in severity and were consistent with those commonly associated with divalproex. A significantly higher proportion of divalproex-treated patients discontinued the study prematurely because of a treatmentemergent adverse event, compared with placebo-treated patients in both the full sample and Cluster B sample. The most common adverse events leading to premature discontinuation of study drug were abnormal laboratory values (eg, increased liver function test values), depression, nausea, and vomiting. The once-daily extended-release formulation of divalproex may potentially improve tolerability in this patient population and deserves study.

Our results in Cluster B patients support those of others who reported decreased impulsive aggressive behavior and irritability in patients treated with divalproex for borderline personality disorder (Hollander et al, 2001a), including those who failed to respond to other antiaggressive agents (ie, SSRIs) (Kavoussi et al, 1998). In a pilot study of women with bipolar II as well as borderline personality disorder, divalproex was superior to placebo for the treatment of irritability and hostility (Frankenburg and Zanarini, 2002). In the current study, patients were excluded if they had bipolar I or bipolar II disorder with recent hypomania (during the past year). Although this suggests that the effect of divalproex in impulsive aggression may be unrelated to its effect in mania, the possibility of a subclinical mood disorder in Cluster B personality disorder cannot be excluded or that impulsive aggression of Cluster B personality disorder has an affective component (see below).

\section{Types and Etiology of Aggression}

The animal literature suggests there may be two broad categories of aggression-affective and predatory (Moyer, 1968; Eichelman et al, 1981; Reis, 1971). Affective aggression is characterized by a high level of arousal and defensive behaviors and is performed in response to a perceived negative outcome. In contrast, predatory aggression involves little physiological arousal and offensive behaviors, and is directed toward a positive reward. In animals, affective forms of aggression have been associated with decreased serotonergic activity and increased noradrenergic and dopaminergic neurotransmission (Eichelman, 1995; Leventhal and Brodie, 1981). Evidence of decreased serotonin activity has been repeatedly reported in individuals with impulsive aggression (Coccaro, 1998a). This finding, as well as those from phenomenological, neuropsychological, and electrophysiological studies, suggests that impulsive aggression is linked more closely to affective aggression (Stein et al, 1995b).

\section{Mechanism of Action}

The mechanism whereby divalproex diminishes impulsive aggression symptoms, as observed in this trial, remains obscure. Although serotonin dysfunction appears important in the etiology of impulsive disorders (Coccaro et al, 1989; Coccaro et al, 1990; New et al, 1997; Virkkunen and Narvanen, 1987; Mann et al, 1992), it is likely that other neurotransmitters also influence impulsive aggression. The inter-related serotonergic, noradrenergic, dopaminergic, opioid, and GABA systems likely contribute, in varying degrees, to impulsivity (Hollander and Evers, 2001b). Divalproex is known to exert effects on GABA, 5-HT, norepinephrine, as well as on limbic kindling (Hollander et $a l, 2002)$, although it is unknown whether any of these mechanisms contribute to the antiaggressive effect found in Cluster B personality disorder. The greater Cluster B responsiveness compared to intermittent explosive disorder and post-traumatic stress disorder may have important implications on how to conceptualize these disorders. For example, it is possible that Cluster B patients may have greater lifetime histories of aggression than the intermittent explosive disorder and post-traumatic stress disorder patients in this study and that such individuals are more likely to respond to divalproex (Kavoussi and Coccaro, 1998). It is also possible that patients with Cluster B personality disorders may share neurobiological features similar to those seen in bipolar spectrum patients who are known to respond to divalproex (Swann et al, 2001). The greater separation between the divalproex and placebo groups that was observed in the Cluster B patients may be based on the effect of divalproex on the affective instability symptom domain in these patients.

\section{Limitations of the Study}

Several factors may have contributed to the negative findings of the overall data, and possibly those in the intermittent explosive disorder and post-traumatic stress disorder subgroups specifically, the most important of which is a considerable placebo response. For the primary end point, a $64 \%$ improvement in median score was observed among patients in the placebo group. This may be attributed, in part, to the positive impact of interaction between patients and clinicians at the weekly visits. An early and substantial placebo effect has also been reported by others in similar patients (Coccaro and Kavoussi, 1997). That the placebo response in Cluster B patients was lower than that in the other diagnostic subgroups may reflect the biologic heterogeneity across the disorders.

Other factors that may have affected the study results include low valproate serum levels and sample size. The recommended mean valproate serum level of $80-120 \mu \mathrm{g} / \mathrm{ml}$ was considered to be a possible therapeutic range based on previous studies (Hollander et al, 2001a). The mean final serum level of $64.2 \mu \mathrm{g} / \mathrm{ml}$ was well below this target. However, this rather medication-naïve population may not have tolerated higher doses or may have required slower titration. This is suggested by the statistically significantly higher discontinuation rate for adverse events with divalproex (17\%) compared to placebo (4\%). Interpretation of results in the post-traumatic stress disorder subgroup is limited by the small sample size. These patients were 
selected for aggressive behaviors and not classic symptoms of post-traumatic stress disorder such as increased arousal, re-experiencing of the traumatic event, and avoidance. The utility of divalproex in patients with post-traumatic stress disorder in general remains to be adequately tested.

There may be substantial heterogeneity of aggressive behaviors and response to treatment among patients with Cluster B personality disorder, intermittent explosive disorder, and post-traumatic stress disorder. Patients enrolled in the study had primarily outwardly directed aggression expressed through verbal assault. While the inclusion criteria did not preclude enrollment of patients with inwardly directed aggression (eg, mutilation, overdose), investigators may have been less likely to enroll such patients because of human subjects concerns as well as the clinical management challenges that accompany such patients. The over-representation of males with outwardly directed aggression in the Cluster B population (63\%) must be considered when making generalizations about the results of this diagnostic subgroup.

The phenomenology of aggression and how it may differ in various psychiatric diagnoses is poorly understood and difficult to evaluate systematically given current evaluation measures. This trial was designed to evaluate impulsive aggression in the absence of a well-established instrument to capture such behaviors. A consensus was reached by experts in impulsive aggression research regarding the inclusion criteria such that the patients could be systematically screened and enrolled. Aggression was measured on a dimensional basis, which is in contrast with the categorical methodology of DSM-IV that is most often used in clinical trials. Although the OAS-M has been used in a number of studies examining impulsive aggression, this assessment quantifies aggressive behaviors (verbal, and assault against others, objects, and self), but does not differentiate between qualitatively different subtypes of aggression (predatory, affective/impulsive). In the current trial, some of the patients enrolled may have had both affective and predatory aggression. Furthermore, change from baseline impulsivity, per se, was not adequately measured by the OAS-M. Future trials should attempt to discriminate between aggression subtypes, as they may have differential responses to pharmacological intervention. Future trials should incorporate specific measurements of impulsivity, perhaps by extracting treatment-sensitive measures of impulsivity from the ADHD literature.

\section{Conclusions}

Impulsive aggression is characterized by significant behavioral disturbances, leading to functional impairment that disrupts social and family relationships and employment, injuries (to self and others), and violent crimes. In spite of the large number of patients with psychiatric disorders who exhibit aggressive behavior and its wide-reaching consequences, consensus is lacking for the optimal treatment approach. No medication is currently approved by the Food and Drug Administration for the treatment of impulsive aggression. A treatment effect was not observed based on the primary end point of this study. However, secondary analyses suggest that divalproex may be an effective agent in treating the symptoms of impulsive aggressive behavior in the Cluster B personality disorder subgroup. Future studies are warranted to investigate the generalizability of these findings to a broader population of patients with Cluster B personality disorder.

\section{ACKNOWLEDGMENTS}

We thank the other investigators, as follows, for their enrollment of patients and participation in this study: Kathleen Brady, MD, Medical University of South Carolina, Charleston, SC; Lori Davis, MD, Tuscaloosa VAMC, Tuscaloosa, AL; James Ferguson, MD, Pharmacology Research Clinic, Salt Lake City, UT; Jeffrey S Kelsey, MD, Emory University, Atlanta, GA; Steven Lindley, MD, National Center for PTSD, Menlo Park, CA; Gopinath Mallya, MD, McLean Hospital, Belmont, MA; John Marshall, MD, Wisconsin Psychiatric Institute and Clinics, Madison, WI; Jeffrey A Mattes, MD, Psychopharm Research, Princeton, NJ; Gerard F Moeller, MD, University of Texas Health Science Center, Houston, TX; George Nurnberg, MD, University of New Mexico Health Sciences Center, Albuquerque, NM; Frederick Petty, MD, Dallas VAMC, Dallas, TX; Mark Rapaport, MD, University of California, La Jolla, CA; Rudolph Steven, DO, San Diego, CA; Christopher Reist, MD, Long Beach VAMC, Long Beach, CA; Carlos Zarate, MD, University of Massachusetts, Worcester, MA; and Mark Zimmerman, MD, Rhode Island Hospital, Providence, RI.

The DSMB members for this study were: Dennis Charney, MD, National Institute of Mental Health, Bethesda, MD; Judith Jones, $\mathrm{MD}, \mathrm{PhD}$, The Degge Group, Ltd, Arlington, VA; James Neaton, PhD, School of Public Health, University of Minnesota, Minneapolis, MN; Larry Siever, MD, Bronx VA Medical Center (116A), Bronx, NY; and Debbie Wentworth, School of Public Health, University of Minnesota, Minneapolis, MN.

Dr Hollander has received research grants from Abbott Laboratories, Bristol Myers Squibb, Eli Lilly and Company, Pfizer Laboratories, Solvay, and Wyeth-Ayerst. He has served as a consultant to and member of the Speakers Bureau of Abbott Laboratories, Solvay, and Wyeth-Ayerst.

Dr Swann has received grant support from Abbott Laboratories, Glaxo SmithKline, UCB Pharma, Bristol Myers Squibb, Eli Lilly, and Shire Laboratories. He has served as a consultant for Abbott Laboratories, Pfizer Laboratories, Shire Laboratories, UCB Pharma, Glaxo SmithKline, Novartis, Eli Lilly, and Bristol Myers Squibb. He has served on Speakers' Bureaus for Abbott Laboratories, Janssen Pharmaceuticals, Novartis, Glaxo SmithKline, and Pfizer Laboratories.

Dr Coccaro is a consultant to Abbott Laboratories and to Eli Lilly Pharmaceuticals. He is a member of the Speakers Bureau for Abbott Laboratories, Eli Lilly Pharmaceuticals, Glaxo SmithKline, and Forest Pharmaceuticals and has received research grants from Abbott Laboratories and Eli Lilly Pharmaceuticals.

Dr McElroy is a consultant to Abbott Laboratories and is a member of the company's Speakers Bureau and Divalproex Advisory Board. She has also received research grants from Abbott Laboratories, and Eli Lilly and Company.

Dr Nemeroff reports the following: Grants/Research: Abbott Laboratories; AstraZeneca; Bristol-Myers-Squibb; Forest Laboratories; Janssen Pharmaceutica; Eli Lilly; 
GlaxoSmithKline; NARSAD; NIMH; Organon; Pfizer Pharmaceuticals; Pharmacia-Upjohn; Stanley Foundation/NAMI; and Wyeth-Ayerst.

Consultant: Abbott Laboratories; Acadia Pharmaceuticals; AstraZeneca; Bristol-Myers-Squibb; Cephalon Pharmaceuticals; Corcept; Cypress Biosciences; Forest Laboratories; GlaxoSmithKline; Janssen Pharmaceutica; Eli Lilly; Merck; Mindsense; Neurocrine Biosciences; Novartis; Organon; Otsuka; Pharmacia-Upjohn; Sanofi; Somerset; Vela Pharmaceuticals; and Wyeth-Ayerst.

Speakers Bureau: Abbott Laboratories; AstraZeneca; BristolMyers-Squibb; Eli Lilly; Forest Laboratories; GlaxoSmithKline; Janssen Pharmaceutica; Organon; Pfizer Pharmaceuticals; and Wyeth-Ayerst.

Stockholder: Corcept and applies only to Dr. Nemeroff

Drs Tracy, Wozniak, and Sommerville are employees of Abbott Laboratories.

\section{REFERENCES}

Allain H, Dautzenberg PH, Maurer K, Schuck S, Bonhomme D, Gerard D (2000). Double blind study of tiapride versus haloperidol and placebo in agitation and aggressiveness in elderly patients with cognitive impairment. Psychopharmacology 148: 361-366.

Barratt ES, Stanford MS, Felthous AR, Kent TA (1997). The effects of phenytoin on impulsive and premeditated aggression: a controlled study. J Clin Psychopharmacol 17: 341-349.

Campbell M, Adams PB, Small AM, Kafantaris V, Silva RR, Shell J et al (1995). Lithium in hospitalized aggressive children with conduct disorder: a double-blind and placebo-controlled study. Am J Acad Child Adolescence Psychiatry 34: 445-453.

Chengappa KN, Ebeling T, Kang JS, Levine J, Parepally H (1999). Clozapine reduces sever self-mutilation an aggression in psychotic patients with borderline personality disorder. J Clin Psychiatry 60: 477-484.

Coccaro EF (1998a). Impulsive aggression: a behavior in search of clinical definition. Harv Res Psychiatry 5: 336-339.

Coccaro E, Gabriel S, Siever L (1990). Buspirone challenge: preliminary evidence for a role of $5 \mathrm{HT} 1 \mathrm{~A}$ receptor function in impulsive aggressive behavior in humans. Psychopharmacol Bull 26: 393-405.

Coccaro EF, Harvey PH, Kupshaw-Lawrence E, Herbert JL, Bernstein DP (1991). Development of neuropharmacologically based behavioral assessments of impulsive aggressive behavior. $J$ Neuropsychiatry Clin Neurosci 3(Suppl): 44-51.

Coccaro EF, Kavoussi RJ (1997). Fluoxetine and impulsive aggressive behavior in personality-disordered subjects. Arch Gen Psychiatry 54: 1081-1088.

Coccaro EF, Kavoussi RJ, Berman ME, Lish JD (1998b). Intermittent explosive disorder revised: development, reliability, and validity of research criteria. Compr Psychiatry 39: 368-376.

Coccaro EF, Siever LJ, Kiar HM, Maurer G, Cochrane K, Cooper TB et al (1989). Serotonergic studies in patients with affective and personality disorders. Correlates with suicidal and impulsive aggressive behavior. Arch Gen Psychiatry 46: 587-599.

Cold JW (1998). Axis II disorders and motivation for serious criminal behavior. In Skodal AE (ed). Psychopathology and Violent Crime. American Psychiatric Press: Washington, DC.

Cowdry RW, Gardner DL (1988). Pharmacotherapy of borderline personality disorder: alprazolam, carbamazepine, trifluoperazine, and tranylcypromine. Arch Gen Psychiatry 45: 111-119.

Donovan SJ, Stewart JW, Nunes EV, Quitkin FM, Parides M, Daniel W et al (2000). Divalproex treatment for youth with explosive temper and mood lability: a double-blind, placebo-controlled crossover design. Am J Psychiatry 157: 818-820.

Donavan SJ, Susser ES, Nunes EV, Stewart JW, Quitkin FM, Klein DF (1997). Divalproex treatment of disruptive adolescents: a report of 10 cases. J Clin Psychiatry 58: 12-15.

Eichelman B (1995). Animal and evolutionary models of impulsive aggression. In Hollander E, Stein DJ (eds). Impulsivity and Aggression. Wiley: Chichester, UK. pp 59-90.

Eichelman B, Elliottt GR, Barchas JD (1981). Biochemical, pharmacological, and genetic aspects of aggression. In: Hamburg DA, Trudeau MB (eds). Behavioral Aspects of Aggression. Alan R. Liss: New York. pp 51-84.

Endicott J, Tracy K, Burt D, Olson E, Coccaro EF (2002). A novel approach to assess inter-rater reliability in the use of the Overt Aggression Scale-Modified. Psychiatry Res 112: 153-159.

Fava M, Rosenbaum JF, Pava JA, McCarthy MK, Steingard RJ, Bouffides E (1993). Anger attacks in unipolar depression, Part 1: clinical correlates and response to fluoxetine treatment. Am Psychiatry 150: 1158-1163.

Fesler FA (1991). Valproate in combat related posttraumatic stress disorder. J Clin Psychiatry 52: 361-363.

First MB, Spitzer RL, Williams JB (1994). Structured Clinical Interview for DSM-IV Axis II Personality Disorders (SCID-II, Version 2.0), Biometric Research, New York State Psychiatric Institute: New York, NY.

Frankenburg FR, Zanarini MC (2002). Divalproex sodium treatment of women with borderline personality disorder and bipolar II disorder: a double-blind, placebo controlled pilot study. J Clin Psychiatry 63: 442-446.

Giakas WJ, Seibyl JP, Mazure CM (1990). Valproate in the treatment of temper outbursts. J Clin Psychiatry 51: 525.

Guy W (1976). ECDEU Assessment Manual for Psychopharmacology, Publication No. ADM 76-336. US Department of Health, Education and Welfare: Rockville, MD.

Haas S, Vincent K, Holt J, Lippman S (1997). Divalproex: a possible treatment alternative for demented elderly aggressive patients. Ann Clin Psychiatry 9: 145-147.

Hollander E, Allen A, Lopez RP, Bienstock CA, Grossman R, Siever LJ et al (2001a). A preliminary double-blind, placebo-controlled trial of divalproex sodium in borderline personality disorder. $J$ Clin Psychiatry 62: 199-203.

Hollander E, Evers M (2001b). New developments in impulsivity. Lancet 358: 949-950.

Hollander E, Posner N, Cherkasky S (2002). Neuropsychiatric aspects of aggression and impulse control disorders. In: Yudofsky SC, Hales RE (eds). American Psychiatric Press Textbook of Neuropsychiatry. American Psychiatric Press: Washington, DC. pp 579-596.

Hori A (1998). Pharmacotherapy for personality disorders. Psychiatry Clin Neurosci 52: 13-19.

Horne M, Lindley SE (1995). Divalproex sodium in the treatment of aggressive behavior and dysphoria in patients with organic brain syndrome. J Clin Psychiatry 56: 430-431.

Itil TM, Wadud A (1975). Treatment of human aggression with major tranquilizers, antidepressants, and newer psychotropic agents. J Nerv Ment Dis 160: 83-99.

Kavoussi RJ, Coccaro EF (1998). Divalproex sodium for impulsiveaggressive behavior in patients with personality disorder. J Clin Psychiatry 59: 676-680.

Khouzam H, Donnelly N (1997). Remission of self-mutilation in a patient with borderline personality disorder during risperidone therapy. J Nerv Mental Dis 185: 348-349.

Leventhal BL, Brodie HKH (1981). The pharmacology of violence. In: Hamburg DA, Trudeau MB (eds). Behavioral Aspects of Aggression. Alan R. Liss: New York. pp 85-106.

Links PS, Steiner M, Boiago I (1990). Lithium therapy for borderline patients: preliminary findings. J Clin Psychopharmacol 4: 173-181. 
Malone RP, Delaney MA, Luebbert JF, Cater J, Campbell M (2000). A double-blind placebo-controlled study of lithium in hospitalized aggressive children and adolescents with conduct disorder. Arch Gen Psychiatry 57: 649-654.

Mann JJ, McBride PA, Anderson GM, Mieczkowski TA (1992). Platelet and whole blood serotonin content in depressed inpatients: correlations with acute and lifetime psychopathology. Biol Psychiatry 32: 243-257.

Moeller FG, Barratt ES, Dougherty DM, Schmitz JM, Swann AC (2001). Psychiatric aspects of impulsivity. Am J Psychiatry 158: 1783-1793.

Moyer KE (1968). Kinds of aggression and their physiological basis. Commun Behav Biol 2: 65-71.

New AS, Trestman RL, Mitropoulou V, Benishay DS, Coccaro E, Silverman J et al (1997). Serotonergic function and self-injurious behavior in personality disorder patients. Psychiatry Res 69: 17-26.

Pattison E, Kahan J (1983). The deliberate self-harm syndrome. Am J Psychiatry 140: 867-872.

Petty F, Davis LL, Nugent AL, Kramer GL, Teten A, Schmitt A et al (2002). Valproate therapy for chronic combat-induced posttraumatic stress disorder. J Clin Psychopharmacol 22: 100-101.

Reis DJ (1971). Brain monoamines in aggression and sleep. Clin Neurosurg 18: 471-502.

Salzman C, Wolfson AN, Schatzberg A, Looper J, Henke R, Albanese $\mathrm{M}$ et al (1995). Effect of fluoxetine on anger in symptomatic volunteers with borderline personality disorder. $J$ Clin Psychopharmacol 15: 23-29.

Sheard M, Marini J, Bridges C, Wagner E (1976). The effect of lithium on impulsive aggressive behavior in man. $A m J$ Psychiatry 133: 1409-1413.

Stein DJ, Simeon D, Frenkel M, Islam MN, Hollander E (1995a). An open-trial of valproate in borderline personality disorder. J Clin Psychiatry 56: 506-510.
Stein DJ, Towey J, Hollander E (1995b). The neuropsychiatry of impulsive aggression. In: Hollander E, Stein DJ (eds). Impulsivity and Aggression. Wiley: Chichester UK. pp 91-105.

Swann AC, Anderson J, Dougherty BM, Moeller FG (2001). Measurement of interepisode impulsivity in bipolar disorder: preliminary report. Psychiatr Res 101: 195-197.

Szymanski HV, Olympia J (1991). Divalproex in posttraumatic stress disorder. Am J Psychiatry 148: 1086-1087.

Tariot PN, Schneider LS, Mintzer JE, Cutler AJ, Cunningham MR et al (2001). Safety and tolerability of divalproex sodium in the treatment of signs and symptoms of mania in elderly patients with dementia: results of a double-blind, placebo-controlled trial. Curr Ther Res 62: 51-67.

Tupin JP, Smith DB, Clanon TL, Kim LI, Nugent A, Groupe A (1973). The long-term use of lithium in aggressive prisoners. Compr Psychiatry 14: 311-317.

Virkkunen M (1975). Reactive hypoglycemic tendency among habitually violent offenders. Nutr Rev 44(Suppl): 94-103.

Virkkunen M, Narvanen S (1987). Plasma insulin, tryptophan, and serotonin levels during the glucose tolerance test among habitually violent and impulsive offenders. Neuropsychobiology 17: $19-23$

Wilcox J (1995). Divalproex sodium as a treatment for borderline personality disorder. Ann Clin Psychiatry 7: 3-7.

Yudofsky SC, Silver JM, Jackson W, Endicott J, Williams D (1986). The overt aggression scale for the objective rating of verbal and physical aggression. Am J Psychiatry 143: 35-39.

Zanarini MC, Frankenburg FR (2001). Olanzapine treatment of female borderline personality disorder patients: a doubleblind, placebo-controlled pilot study. J Clin Psychiatry 62: 849-854. 